\title{
Assessment of an in vitro whole cigarette smoke exposure system: The Borgwaldt RM20S 8-syringe smoking machine
}

\author{
Jason Adamson, David Azzopardi, Graham Errington, Colin Dickens, John McAughey and Marianna D Gaça*
}

\begin{abstract}
Background: There have been many recent developments of in vitro cigarette smoke systems closely replicating in vivo exposures. The Borgwaldt RM20S smoking machine (RM20S) enables the serial dilution and delivery of cigarette smoke to exposure chambers for in vitro analyses. In this study we have demonstrated reliability and robustness testing of the RM20S in delivering smoke to in vitro cultures using an in-house designed whole smoke exposure chamber.

Results: The syringe precision and accuracy of smoke dose generated by the RM20S was assessed using a methane gas standard and resulted in a repeatability error of $\leq 9 \%$. Differential electrical mobility particle spectrometry (DMS) measured smoke particles generated from reference 3R4F cigarettes at points along the RM20S. 53\% $\pm 5.9 \%$ of particles by mass reached the chamber, the remainder deposited in the syringe or connecting tubing and $\sim 16 \%$ deposited in the chamber. Spectrofluorometric quantification of particle deposition within chambers indicated a positive correlation between smoke concentration and particle deposition. In vitro airliquid interface (ALI) cultures (H292 lung epithelial cells), exposed to whole smoke (1:60 dilution (smoke:air, equivalent to $\left.\sim 5 \mu \mathrm{g} / \mathrm{cm}^{2}\right)$ ) demonstrated uniform smoke delivery within the chamber.

Conclusions: These results suggest this smoke exposure system is a reliable and repeatable method of generating and exposing ALI in vitro cultures to cigarette smoke. This system will enable the evaluation of future tobacco products and individual components of cigarette smoke and may be used as an alternative in vitro tool for evaluating other aerosols and gaseous mixtures such as air pollutants, inhaled pharmaceuticals and cosmetics.
\end{abstract}

\section{Background}

Molecular mechanisms and pathways elucidating cigarette smoking disease processes are still not well understood. Abundant epidemiological studies and research have linked smoking to a number of diseases including lung cancer, chronic obstructive pulmonary disease and cardiovascular disease; identified inflammatory and oxidant stress mechanisms having a pivotal role in all of these processes [1]. Physiologically-relevant in vitro model systems, in which human lung cells and tissues can be exposed to appropriate doses of cigarette smoke may provide useful tools to interpret these processes and identify other mechanisms. There are numerous studies reporting the development of such in vitro

* Correspondence: Marianna_Gaca@bat.com

British American Tobacco, Group R\&D, Regents Park Road, Southampton, SO15 8TL, UK models utilising a variety of cell types and systems [2-8]. However, just as the selection and cultivation of these selected cells and systems are important, so too is the development, generation and delivery of cigarette smoke to these in vitro assays.

Traditionally, toxicological evaluation of cigarette smoke has focussed on the particulate fraction of smoke. The particulate matter (PM) from cigarette smoke is collected on a Cambridge filter pad [9] and eluted with an organic solvent such as methanol or dimethyl-sulphoxide to generate a solution $[10,11]$ which can be easily added to traditional submerged in vitro cultures. This fraction of cigarette smoke only contains the particulate element, which constitutes approximately $5-10 \%$ of cigarette smoke by weight. The remaining $90-95 \%$ constitutes the gas/vapour phase of smoke and is not captured on the Cambridge filter pad 
[12]. Water-soluble cigarette smoke components from both particulate and vapour phases can be captured by bubbling cigarette smoke through biological buffers or tissue culture media. This resulting 'aqueous extract' can also be easily assessed in submerged in vitro cultures [13-15]. However, in order to understand the toxicological and biological effect of cigarette smoke, the whole cigarette smoke, i.e., both particulate and vapour phase, needs to be analysed.

Recently there has been an increase in the development of novel whole smoke exposure systems to generate and deliver whole cigarette smoke to in vitro cellular culture systems for toxicological and biological assessment [16]. There are many smoke machines commercially available including systems such as the Borgwaldt RM20S (RM20S) smoking machine [7,17-19], the Burghart Mimic Smoker-01 ${ }^{\circledR}[20]$ and the Vitrocell ${ }^{\circledR}$ VC $10^{\circledR}$ Smoking Robot [21]. There are also several cell exposure chambers/systems described in the literature including CULTEX ${ }^{\circledR}$ modules [22] the rocking platform system [23] and British American Tobacco's (BAT) exposure chamber (Figure 1) [7,17]. These exposure systems are of greater physiological relevance compared to traditional submerged exposure methods as they support cell cultures cultured at the air-liquid interface (ALI) and enable the exposure of cells to both phases of smoke simultaneously, capturing any synergies or interactions between the two. Despite these advances, there have been few studies to characterise cigarette smoke in these systems [20].

At BAT we are investigating the use of a number of these smoking machines, including the RM20S smoking machine (Figure 2). The RM20S generates, dilutes and delivers whole smoke to in vitro cultures placed in an exposure chamber. Serial dilutions are made via multiple steps of filling and partial emptying of the syringe once filled with smoke and air. The exposure chamber houses cell inserts, such as commercially available Transwells ${ }^{\circledR}$, providing a porous support for ALI cultures to be dosed apically with cigarette smoke and for warmed cell culture media to be delivered basally. The RM20S is commercially supplied as a four syringe smoking machine and has been previously assessed for robustness of smoke delivery [18].

In conjunction with Borgwaldt, we have expanded the capability of the smoke delivery system with the addition of another four syringe unit. This addition has resulted in a new expanded eight syringe system, requiring characterisation which has not been conducted previously. Adapting the system from four to eight syringes increases the number of available whole smoke doses per run and reduces the number of experimental runs (eliminating the need for back-to-back runs) which results in a single biological dose response to be carried out in one experimental run. This adaptation will enhance the RM20S by increasing flexibility in experimental design, by reducing inter-run variability and by saving time.

The aim of this study was to characterise and evaluate the performance of the expanded RM20S eight syringe smoking machine and to assess cigarette smoke through the system. Initially all syringes were calibrated for precision and accuracy. Using a hydrocarbon analyser and test gas the syringes could be tested to confirm to what extent, collectively and individually, smoke was being delivered at its target dilution. The penetration and deposition of cigarette smoke particulate throughout the RM20S whole smoke exposure system was investigated using two dosimetry techniques. The first employed the use of differential electrical mobility particle spectrometry (DMS) to enable in-line and real-time particle diameter and concentration measurement of smoke along

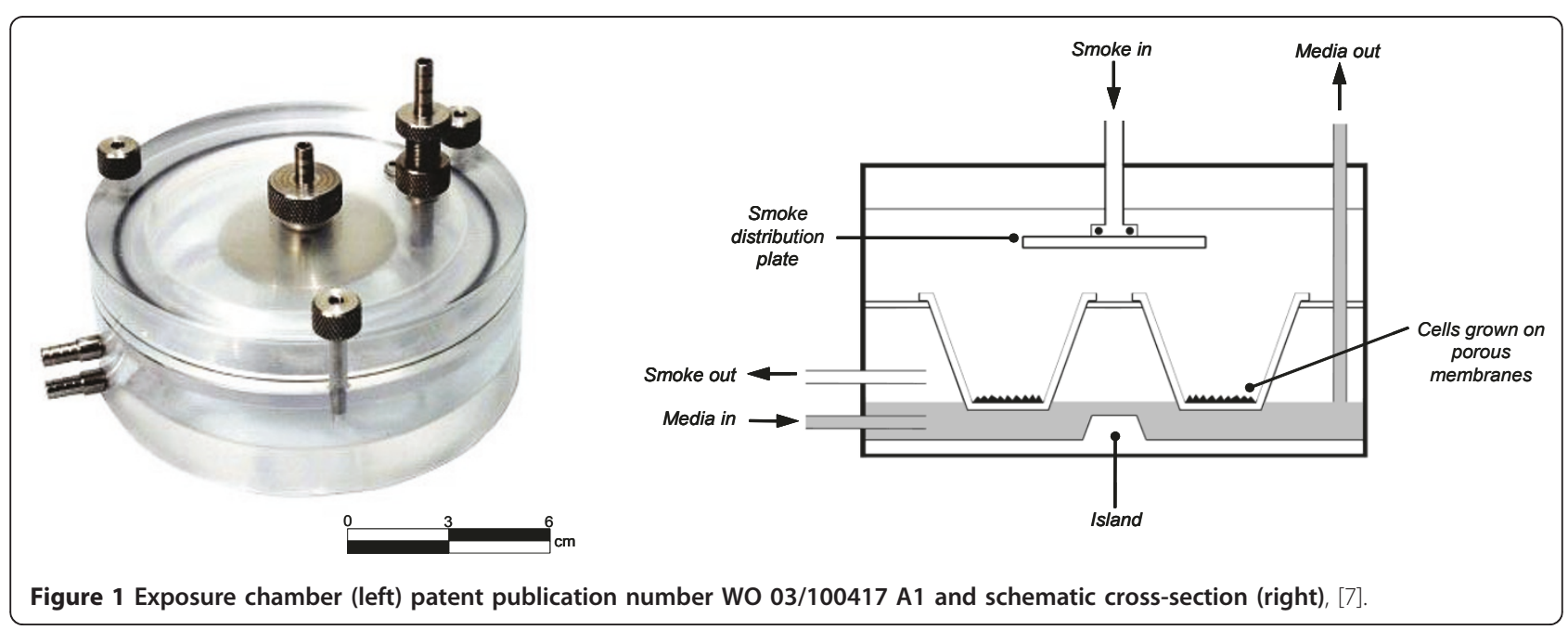




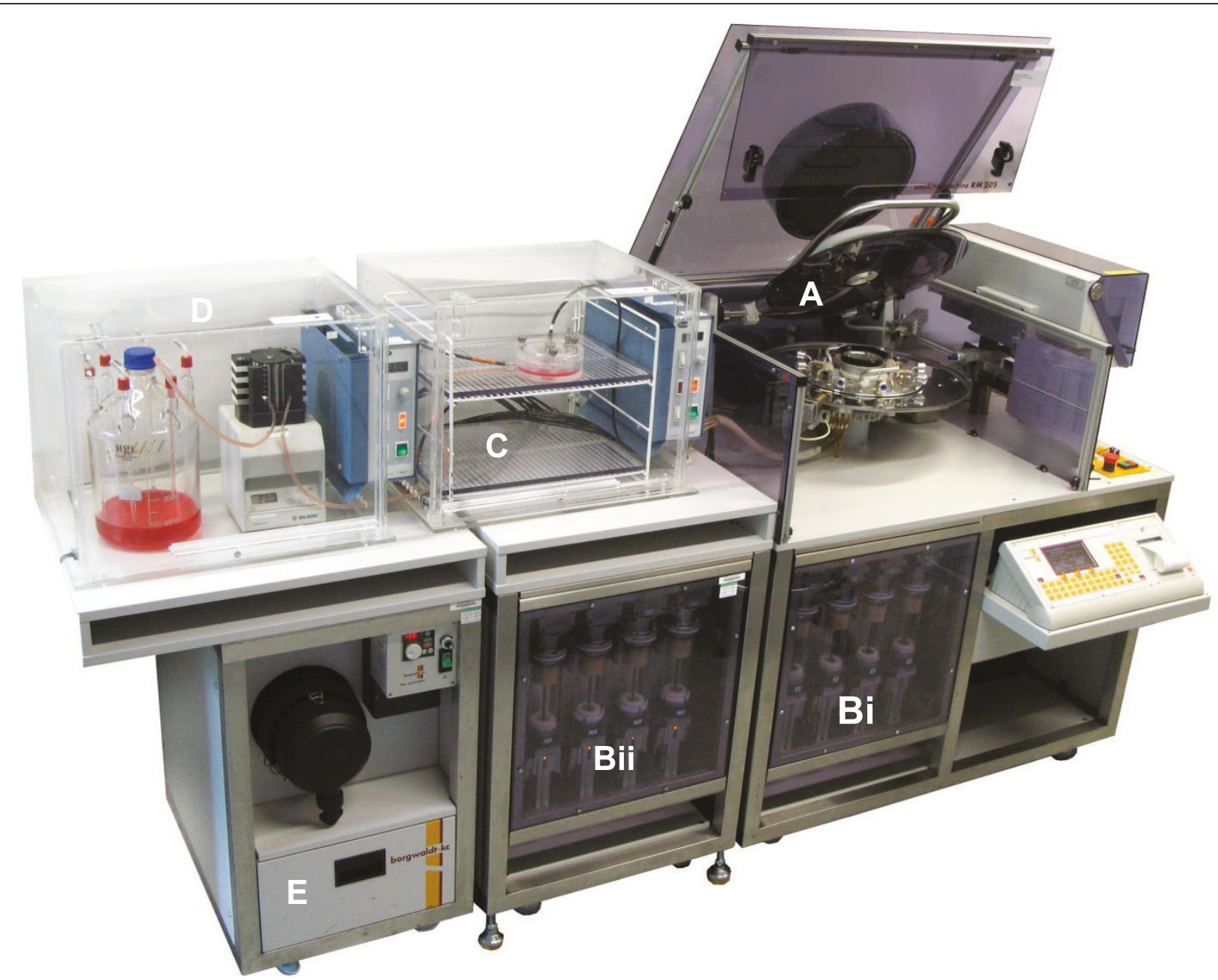

Figure 2 The Borgwaldt RM20S 8 syringe smoking machine. The dilutor syringes work independently, with the capability of delivering an extensive smoke dilution range (1:2 -1:4,000 [smoke:air, v/v]) and delivering up to eight different doses of whole smoke simultaneously. A cigarette smoke generator; $\mathbf{B i}$ - original 4-syringe unit; Bii - additional 4-syringe unit enabling 8 concurrent dilutions in a single run; $\mathbf{C}$ - a single BAT exposure chamber housed in an incubator at $37^{\circ} \mathrm{C}$ attached to smoke generator and media (up to 9 chambers can be installed in the incubator shown, one connected to each syringe plus an air control); $\mathbf{D}$ - cell culture media maintained at $37^{\circ} \mathrm{C}$ in an incubator, supplied to chambers using a pump; $\mathbf{E}$ - airflow fan controller.

the whole smoke system [24]. It had been previously reported by Scian et al., (2009) that the Burghart Mimic Smoker- $01^{\circledR}$ system has smoke losses of up to $50 \%$ preceding delivery to cells [20]. Data generated from the DMS would enable us to calculate smoke losses experienced in our exposure system and compare them to this recent study. The second technique used spectrofluorometric analysis to quantify particulate deposition on inserts within the exposure chamber during whole smoke exposures. Particulate is extracted followed by absorbance measurements which can be converted into concentrations using a PM standard curve. Finally, to confirm previous non-cell based methods [17] using cell cytotoxicity, positional exposure chamber dosimetry was examined to ensure the same dose of cigarette smoke was being delivered to each insert within a single chamber. H292 lung epithelial cells were cultured on individual inserts within an exposure chamber, exposed to whole smoke and cytotoxicity assessed using the Neutral Red Uptake (NRU) assay.

Results from this study have given us further confidence that the current whole smoke exposure system, using the Borgwaldt RM20S smoking machine and the BAT exposure chamber, is a reliable, repeatable, robust and potentially a more physiologically relevant method of generating and exposing in vitro cellular and tissue cultures to whole cigarette smoke at the ALI. We propose to further characterise whole smoke dosimetry relative to specific smoke constituents and to use this system for the evaluation of novel cigarette design 
modifications proposed to reduce toxicants in smoke and to further investigate individual components of cigarette smoke.

\section{Results}

\section{Syringe validation using hydrocarbon analysis}

Initially, before conducting any in vitro whole smoke exposures with the RM20S, all eight syringes were calibrated for precision and accuracy using a methane standard gas. Using a hydrocarbon analyser and methane test gas at two dilutions (1:193 and 1:500 (test gas:air, volume:volume)), the syringes, individually and collectively, were assessed to determine if they were achieving the target deliveries of $520 \pm 20 \mathrm{ppm}$ and $200 \pm 15 \mathrm{ppm}$ methane gas standard respectively.

At a dilution of 1:193 individual syringe precision suggested no bias of average syringe outputs (Figure 3A). All

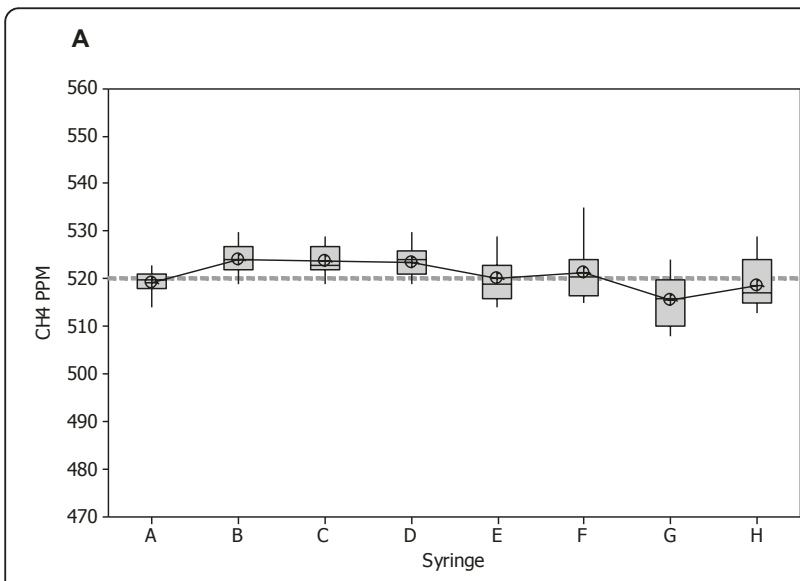

B

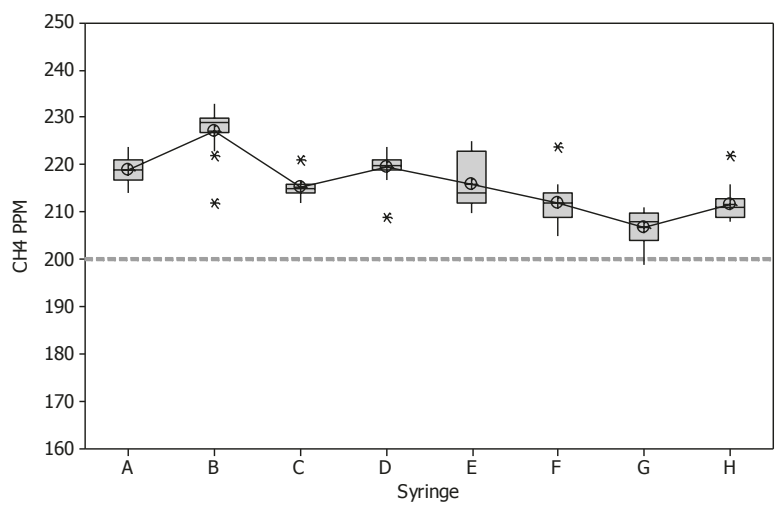

Figure 3 The precision of eight individual syringes using hydrocarbon analysis. A methane gas standard was diluted by the eight Borgwaldt RM20S syringes and precision was calculated as a function of accuracy of dilution delivery. A - syringe precision at a dilution of 1:193 (smoke:air, v/v) against a target value of $520 \mathrm{ppm}$ methane gas standard, $n=15$. B - syringe precision at a dilution of 1:500 (smoke:air, v/v) against a target value of 200 ppm methane gas standard, $n=15,{ }^{*}=$ outliers. eight syringes delivered with little variability at the target level of $520 \pm 20 \mathrm{ppm}$. Repeatability, which is an average of how individual syringes are performing as a percentage of the nominal values, was calculated at $2.3 \%$. Collective syringe performance was assessed using the Repeatability and Reproducibility (Gage R\&R) estimate, which indicates consistency and stability of syringe performance across the entire system, and as a percentage of the nominal values was $3 \%$. All of these R-values were $<10 \%$, which are generally accepted limits in measurement systems indicating the equipment is fit for purpose [25].

At a dilution of 1:500, individual syringe precision indicated a $15 \%$ positive bias of average syringe outputs about the target $(200 \pm 15 \mathrm{ppm})$ (Figure 3B). Dunnet's test showed that collectively the syringes were significantly different from the target. Repeatability, as a percentage of the nominal values, was $5 \%$ and the Gage $\mathrm{R} \& \mathrm{R}$ estimate as a percentage of the nominal values was $8.9 \%$. Although bias was significant, it only accounts for the overall position of the data and not the precision of syringe delivery, therefore bias can be significant yet the $R \& R$ estimate be fit for purpose within the same scenario. All of these values were $<10 \%$, and hence deemed to be fit for purpose despite significant bias.

A two-way ANOVA was conducted to assess the syringe outputs across both dilutions and overall accuracy. At the dilutions measured there was no significant difference in the syringes $(\mathrm{p}=0.051)$. Total Gage $\mathrm{R} \& \mathrm{R}$ was $2.9 \%$, reproducibility was $2.2 \%$ and repeatability was $1.9 \%$ for the entire syringe system at both dilutions tested. These results show that collectively the syringe system is fit for purpose.

\section{Smoke transfer efficiency using an electrical mobility spectrometer}

The penetration and deposition of particulate throughout the RM20S whole smoke exposure system was investigated using an electrical mobility spectrometer (DMS). The DMS sampled whole smoke at the exit of the syringe after the primary dilution $(\alpha)$, pre-entry to the chamber $(\beta)$ and at the exit from the chamber $(\gamma)$, shown in Figure 4. Additionally, a three-way comparison of different tubing types was conducted; the types tested were existing polyurethane (the tubing installed in the RM20S upon manufacture and having a visible build up of tar), unused polyurethane and unused Viton ${ }^{\circledR}$, a PTFE-like fluoroelastomer (non-stick).

Particle penetration was calculated by both particle mass and number, by comparing the total smoke particulate at three points. The values were reported relative to $100 \%$ smoke mass equivalent to $5.75 \pm 0.31 \mathrm{mg}$ (assuming unit density), and $100 \%$ smoke particle number equivalent to $1.44 \times 10^{12} \pm 0.10 \times 10^{12}$ particles (n $=3$ ) for 10 puffs from 2 cigarettes $(6+4)$ as measured by 


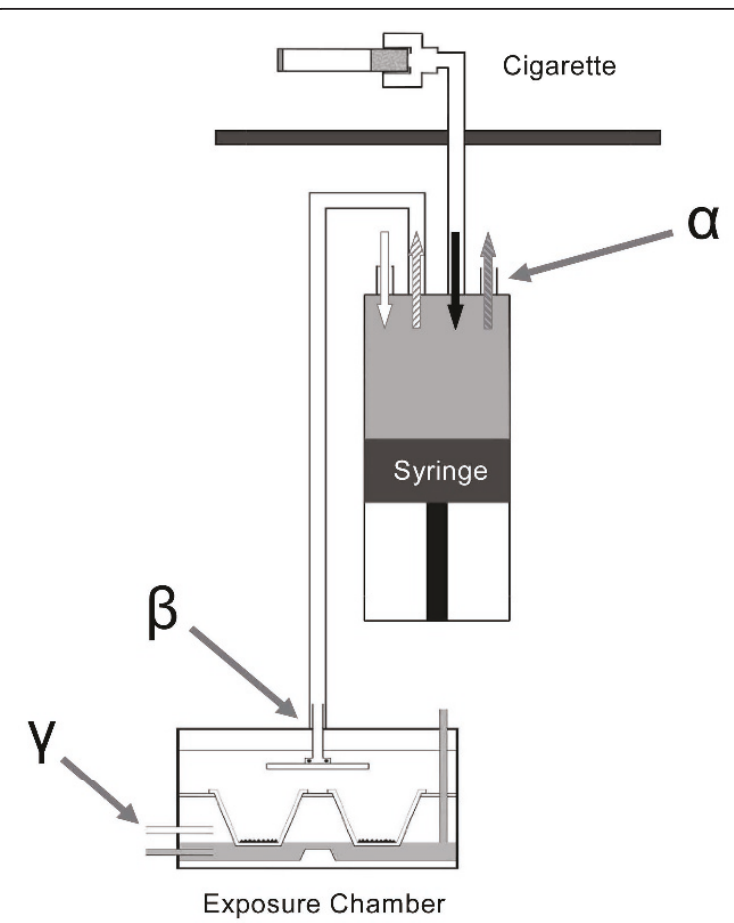

Arrows indicate the serial dilution process within the syringe: ISO puff of mainstream cigarette smoke $\longrightarrow$; sterile air for serial dilutions $\Longrightarrow$; waste exhaust from serial dilutions $\Longrightarrow$; final dilution of smoke delivered to chamber $\rightleftarrows$.

Figure 4 Schematic cross-section of a single RM20S syringe and exposure chamber. Smoke was sampled at the exit of the syringe $(\alpha)$, pre-entry to chamber $(\beta)$ and at the exit from the chamber $(\gamma)$ using an electrical mobility spectrometer.

the DMS-smoke cycle simulator (SCS) system pre-dilution at the point of generation. This matches the 10 puff data collection at the other sample points. Data was corrected for the theoretical delivered fraction at each sampling point as calculated based on the dilution settings and mechanism. Pre-chamber losses were calculated by the difference in smoke concentration between the point of smoke generation $(100 \%)$ and the chamber $(\beta)$, and chamber deposition was calculated by the difference in smoke concentration before $(\beta)$ and after $(\gamma)$ the chamber.

As shown in Figure 5, very similar DMS dosimetry results were achieved using the different tubing types, suggesting that the choice of tubing type did not matter for these three; there was no statistically significant difference between the three types tested, $\mathrm{p}=0.083$, but there was significant difference between the three sampling points $\alpha, \beta$ and $\gamma$ for all three, $\mathrm{p}=<0.05$. Pre-syringe losses based on particle mass for existing polyurethane were $14 \%$, with $86 \%$ particle mass being detected at the exit of the syringe (a). Pre-chamber losses were $47 \%$ in total, $33 \%$ of

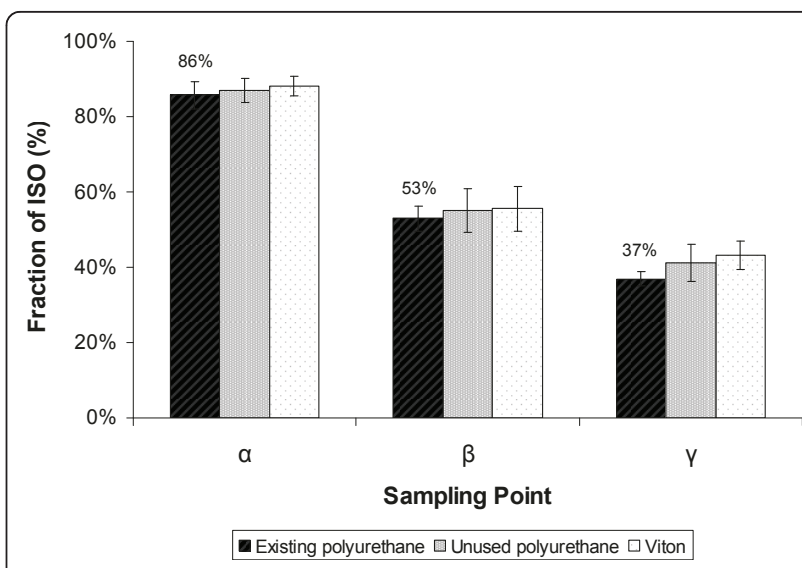

Figure 5 Assessment of cigarette smoke particle mass penetration through the RM20S system, using three different tubing types. An electrical mobility spectrometer sampled smoke at the exit of the syringe $(\alpha)$, before the chamber $(\beta)$ and at the exit from the chamber $(\gamma)$. Results expressed as percentage of average mainstream smoke $(100 \%=5.75 \pm 0.31 \mathrm{mg}) \mathrm{n}=3$. Statistically significant differences were observed between the three sampling points $(\alpha, \beta, \gamma)$ for all tubing types tested, $p=<0.05$, but no significant differences were observed between the three tubing types at each individual sampling point, $p=0.083$.

which was lost/deposited within the syringe and connecting tubing (the difference between $\alpha$ and $\beta$ ), with $53 \%$ average particle penetration to the chamber. Chamber particle deposition by mass was $16 \%$, but it must be noted that this was a mixture of fresh and old smoke.

Table 1 summarises all the data generated from existing polyurethane only (as there was no significant differences between the three types tested, and existing

Table 1 Summary of the assessment of total cigarette smoke particulate sampled at the exit of the syringe $(\alpha)$, before the chamber $(\beta)$ and at the exit from the chamber $(\gamma)$ using an electrical mobility spectrometer; existing polyurethane was the tubing installed

\begin{tabular}{lccc}
\hline & $\boldsymbol{\alpha}$ & $\boldsymbol{\beta}$ & $\boldsymbol{\gamma}$ \\
\hline Particle mass penetration (\%) & 86 & 53 & 37 \\
& \pm 3.2 & \pm 5.9 & \pm 4.9 \\
Particle number penetration (\%) & 14.7 & 10.3 & 8.3 \\
& \pm 0.5 & \pm 0.6 & \pm 0.7 \\
Count Median Diameter (nm) & 301.0 & 293.9 & 289.1 \\
& \pm 1.1 & \pm 5.8 & \pm 5.8 \\
Volume Median Diameter (nm) & 422.9 & 393.8 & 360.8 \\
& \pm 2.8 & \pm 10.4 & \pm 8.9 \\
\hline
\end{tabular}

Particle penetration was calculated by both particle mass and number, by comparing the total smoke particulate to the sample points $\alpha, \beta$ and $\gamma$ and reported relative to $100 \%$ smoke mass equivalent to $5.75 \pm 0.31 \mathrm{mg}$ (assuming unit density) and 100\% smoke particle number equivalent to $1.44 \times$ $1012 \pm 0.10 \times 1012$ particles $(n=3)$. Significant differences were observed between all sampling points for particle mass, number and VMD, and for CMD between the $\alpha$ and $\gamma$ sampling points $(p=<0.05)$; there were no significant differences for CMD between $\alpha$ and $\beta(p=0.0549)$, and between $\beta$ and $\gamma$ (0.7384). 
polyurethane remains in the machine as the preferred type). Percentage of particle number penetration was lower than particle mass penetration and this is likely due to coagulation; particle mass is conserved but particle number reduces as particles collide and coalesce during early transit. However, preferential diffusional and evaporative losses of smaller particles within the distribution are also feasible. The reductions in Count Median Diameter (CMD) were not as pronounced as the reductions in Volume Median Diameter (VMD) at the three points tested, and this suggests that larger particles were depositing preferentially in the tubing and syringe.

Pairwise comparison using the General Linear Model showed that there were statistically significant differences $(p=<0.05)$ between all three sampling points in terms of particle number, particle mass and VMD. However, for CMD there was only a significant difference between the $\alpha$ and $\gamma$ sampling points; for CMD there was little difference between $\alpha$ and $\beta(\mathrm{p}=0.0549)$, and between $\beta$ and $\gamma$ (0.7384).

\section{Deposition assessment using fluorometric analysis}

Particulates from 3R4F reference cigarettes (10 mg tar) deposited onto the inserts in the exposure chamber during whole smoke exposures were eluted, quantified using fluorescence spectroscopy and converted to $\mu \mathrm{g} /$ $\mathrm{cm}^{2}$ using a PM standard curve. The particulate deposition data was plotted onto a calibration graph (insert deposition against corresponding Borgwaldt RM20S smoke dilution) using GraphPad Prism version 5.02. Both axes were logged and a linear regression created (Figure 6).

The linear range of the graph was over 2 orders of magnitude for smoke dilution (1:5 - 1:400 (smoke:air, v/ v)) with particle mass deliveries of $60.74-0.63 \mu \mathrm{g} / \mathrm{cm}^{2}$. The Limit of Quantification (LOQ) and Limit of Detection (LOD) were calculated to be 0.448 and $0.247 \mu \mathrm{g} /$ $\mathrm{cm}^{2}$ respectively. The $\mathrm{R}$-square value for the linear

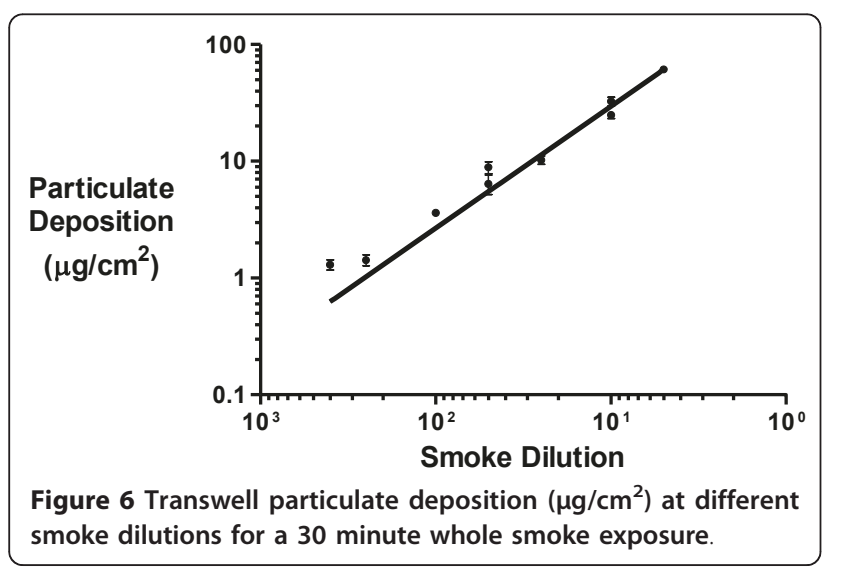

regression was 0.916 . As expected, the data showed that there was a positive correlation between smoke concentration and particulate depositing on the inserts.

The calibration graph enabled the interpolation of either an insert particulate concentration from a desired smoke dilution or vice versa. The linear regression can be used to generate the following table (Table 2) displaying the equivalent insert particulate deposition values $\left(\mu \mathrm{g} / \mathrm{cm}^{2}\right)$ for a specific Borgwaldt RM20S smoke dilution.

\section{Exposure chamber dosimetry}

Cell cytotoxicity, using the NRU assay was used to assess individual insert whole smoke dose within a single chamber, to investigate positional exposure chamber dosimetry. Cell viability was calculated as a percentage of the air controls (as described in the methods) and incubator controls were included in every run to use as a comparator/indicator of cell stress and sterility. Mean viability across all six inserts was $46 \pm 9.8 \%(\mathrm{p}=0.56, \mathrm{n}$ $=20$ ). Figure 7 indicates there was no significant difference between individual inserts exposed to cigarette smoke in a single exposure chamber, suggesting uniform smoke distribution in the chamber. The data range however suggests that the inter-run variability is large within each individual Transwell $^{\circledR}$ rather than intra-run variability.

\section{Discussion}

In this study we present a characterised and evaluated system used to generate, dilute, deliver and expose in vitro cell cultures to whole smoke at the ALI. The integrity of whole smoke generated and delivered to in vitro cultures was assessed using a measurement systems analysis approach. We characterised three key areas within the exposure system; delivery and accuracy of dilution syringes, smoke particle penetration and smoke losses

Table 2 Summary of calculated 3R4F particulate deposition on inserts within the exposure chamber $\left(\mu \mathrm{g} / \mathrm{cm}^{2}\right)$

\begin{tabular}{|c|c|}
\hline $\begin{array}{l}\text { Smoke dilution } \\
\text { (smoke:air, v/v) }\end{array}$ & Equivalent 3R4F particulate deposition $\left(\mu \mathrm{g} / \mathrm{cm}^{2}\right)$ \\
\hline $1: 5$ & $60.74 \pm 2.07$ \\
\hline $1: 10$ & $29.47 \pm 1.30$ \\
\hline $1: 25$ & $11.33 \pm 0.59$ \\
\hline $1: 50$ & $5.50 \pm 0.58$ \\
\hline $1: 100$ & $2.67 \pm 0.13$ \\
\hline $1: 250$ & $1.03 \pm 0.11$ \\
\hline $1: 400$ & $0.63 \pm 0.09$ \\
\hline
\end{tabular}

Chambers were exposed to various whole smoke dilutions during a 30 minute exposure to 3R4F reference cigarettes smoked at the ISO regime. Particles deposited on inserts were measured by fluorescence detection. Deposited particulate matter was eluted with a solvent and quantified against a standard curve produced from 3R4F cigarette smoke total particulate matter. 


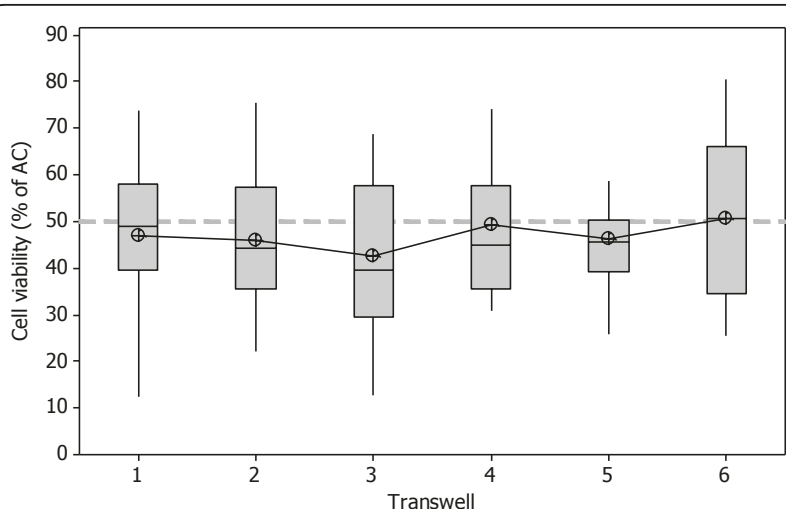

Figure 7 Exposure chamber dosimetry investigated using NRU; cytotoxicity in $\mathrm{H} 292$ cells exposed to a smoke dilution of 1:60 (smoke:air, v/v) in $12 \mathrm{~mm}$ Transwells ${ }^{\circledR}$ in a single exposure chamber. Dotted line represents $50 \%$ viability $\left(E_{50}\right)$. Mean viability $=46 \pm 9.8 \%, p=0.56, n=20$.

through the system, and positional deposition in the exposure chamber.

Individual syringe precision and accuracy were measured using the hydrocarbon analysis method described. The syringes were tested at two dilutions, 1:500 and 1:193 (a calibration standard dilution), as this represents the extreme top and mid dilutions respectively used in the laboratory to generate a biological dose response range using robust in vitro primary and continuous cell cultures $[7,17]$. We observed more variation about the higher dilution of 1:500, target value of $200 \mathrm{ppm}$ methane gas, when compared to the lower dilution of 1:193, target value of $520 \mathrm{ppm}$ methane gas; this was primarily because the syringe sensors were adjusted at the latter dilution. At a dilution of 1:500 there was a positive bias about the target of $15 \%$, suggesting at this dilution, collectively the syringes were delivering $15 \%$ more gas, and could therefore deliver $15 \%$ more smoke. This is something which cannot currently be improved upon, as bias fluctuates at different dilutions tested, but this can be adjusted for with further syringe calibration.

Furthermore, earlier evaluations from data taken at four dilution levels (1:1000, 1:500, 1:250, 1:193) demonstrated that both repeatability and the Gage R\&R estimate improved as the dilution was decreased (smoke concentration increased) towards the biological dose response range previously described (data not shown). This may be due to the reduction in serial dilutions the syringe has to make as smoke concentration increases, and therefore eliminating additional stages of potential error. At a dilution of 1:193 there was no bias of syringe delivery about the target and the Gage R\&R estimate was $3 \%$. Statistical analysis indicated no significant differences in the syringes and Total Gage R\&R to be within acceptable limits $(<10 \%)$. These results have demonstrated that within the biological dose response range, individual syringe precision and accuracy of whole smoke dilutions are fit for purpose which suggests confidence in the programmed dilution of smoke generated by the RM20S. This study is further supported by a previous RM20S inter-laboratory study by Kaur et al., (2010) comparing syringe validation using $\mathrm{CH}_{4}$ measurements; in two different laboratories and with two different operators there was comparable reproducibility and precision for commonly used whole smoke dilutions [18].

For whole smoke exposure systems in general, it is important to understand smoke losses and smoke changes between the point of generation and exposure chamber and smoke deposition within the chamber itself. Looking only at the existing polyurethane (preferred tubing), average particle penetration to the chamber was $53 \%$ by mass, implying that there are losses (about 47\%) within the syringe or within the tubing between the syringe and the chamber. This is also consistent with the Burghart Mimic Smoker-01 ${ }^{\circledR}$ system which reported smoke losses of up to $50 \%$ preceding delivery to cells [20]. Repeat studies indicated smoke penetration was very consistent in the RM20S, so smoke delivered to the chamber will be consistent at this dilution setting of 1:60 (smoke:air, v/v).

Particle penetration by number to each of the sampling points was significantly less than particle mass. It is the loss of particle number prior to $\alpha$ that is important; this is assumed to be caused by significant predilution coagulation of particles resulting in reduced particle number ( $14 \%$ reduction in number). The percentage particle loss by number and mass between sample point $\alpha$ and sample point $\beta$ was relatively consistent; $30 \%$ number and $40 \%$ by mass lost (relative). Additionally the VMD at sample point $\beta(393.8 \mathrm{~nm})$ was less than at sample point $\alpha(422.9 \mathrm{~nm})$. These imply that larger particles are preferentially being lost in the syringe/tubing. Therefore it is likely that most of the observed losses occurred in the syringe during the generation of serial dilutions of smoke and turbulent airflow, and there is evidence for this in the rapid visual build-up of tar on the glass surface during frequent usage.

Furthermore we investigated the use of other tubing types in the RM20S system: existing polyurethane (used tubing, currently installed in the RM20S and having a visible build up/conditioning of tar), unused polyurethane and unused Viton ${ }^{\circledR}$, a PTFE-like non-stick fluoroelastomer. Identical DMS dosimetry studies using different tubing types, including the Viton ${ }^{\circledR}$, suggest that the choice of tube material did not matter for these three. 
To complement the DMS dosimetry studies, we have also assessed particulate deposition using high performance liquid chromatography. Particulate, generated from different whole smoke doses from reference 3R4F cigarettes (10 mg tar), captured on blank inserts were collected, eluted, and quantified in $\mu \mathrm{g} / \mathrm{cm}^{2}$ using a PM standard curve. A reference table was generated from the data obtained to allow comparisons between particulate deposition and whole smoke dilution. We have conducted similar studies using other cigarette types with different tar deliveries $(6 \mathrm{mg}$ and $1 \mathrm{mg}$ ISO pack tar compared with 3R4F $10 \mathrm{mg}$ ISO pack tar). Data indicated there was a clear difference in particulate depositing on the inserts from each of the different cigarette types, with a greater amount of particulate depositing from the highest delivery $10 \mathrm{mg} 3 \mathrm{R} 4 \mathrm{~F}$ product, followed by the $6 \mathrm{mg}$ product which in turn is greater than the $1 \mathrm{mg}$ product. For example, at a 1:5 smoke dilution (smoke:air, v/v) generated by the RM20S, particulate delivery was $61.05 \pm 2.07$, $38.11 \pm$ 2.21 and $3.09 \pm 0.21 \mu \mathrm{g} / \mathrm{cm}^{2}$ for the 10,6 and $1 \mathrm{mg}$ products respectively.

Finally, exposure chamber dosimetry was assessed by measuring cell viability within a single chamber, and comparing cell inserts which were exposed to the same dilution of cigarette smoke. Previous studies on the Borgwaldt RM20S and exposure chamber using chemical analysis techniques reported particulate deposition in the chamber to be uniform [17] however this had never been tested before using a biological endpoint. Cell viability assessed using the NRU assay was used as a quick and robust method to test for differences in doses between cell inserts. As verified by a p-value of 0.56 , there was no significant difference in cell viability at the dose tested within a single chamber, demonstrating that smoke/cell monolayer interactions were equal across all inserts within a chamber (mean viability $46 \pm 9.8 \%$ ).

\section{Materials and methods}

\section{Cell culture and whole smoke exposure}

Human pulmonary carcinoma cells (NCI-H292) from the American Type Culture Collection (ATCC; passages 90-94) were cultured in complete RPMI cell culture medium and incubated at $37^{\circ} \mathrm{C}, 5 \% \mathrm{CO}_{2}$. Cells were seeded in $12 \mathrm{~mm}$ diameter, $0.4 \mu \mathrm{m}$ pore size inserts (Transwell $^{\circledR}$, Corning, Schiphol-Rijk, The Netherlands) 2 days prior to exposure, at a density of $3 \times 10^{5}$ cells $/ \mathrm{ml}$. Cells were exposed to mainstream cigarette smoke at the air-liquid interface, using the BAT exposure chamber as previously described (Figure 1) [7,17]. In brief, six inserts were placed in a single exposure chamber and exposed to cigarette smoke for 30 minutes at a dilution of 1:60 (smoke:air, $\mathrm{v} / \mathrm{v}$ ); this is approximately the half maximal effective concentration $\left(\mathrm{EC}_{50}\right)$ of $3 \mathrm{R} 4 \mathrm{~F}$ cigarette whole smoke to induce $50 \%$ cytotoxicity in exposed H292 cells, as determined by previous experiments [17].

Air and incubator controls were also included in each run. Air control cells were exposed to a continuous flow of sterile air in an exposure chamber, at $200 \mathrm{~cm}^{3} / \mathrm{min}$ for a 30 minute duration, at the same time as whole smoke exposed cells. Incubator control cells remained in submerged condition in cell culture incubators throughout the exposures.

\section{Cigarette smoke generation}

3R4F reference cigarettes (10 mg ISO pack tar) (University of Kentucky, Kentucky, USA) were smoked at a programmed dilution of 1:60 (smoke:air, v/v) according to ISO 4387:1991 [10] (35 ml puff over 2 seconds, once a minute), as previously described [17]. Between puffs, smoke was allowed to remain in the exposure chamber and was only expelled passively when the next puff replaced it. Allowing smoke to age during the deposition phase of the cycle in this exposure system differs from alternative flow-through systems for smoke exposure reported elsewhere [20-22]. The rationale in this case was to increase relative deposition efficiency and improve precision of deposition by allowing diffusion and sedimentation from still air in a symmetrical exposure chamber. The 3R4F cigarettes were conditioned for a minimum of 72 hours prior to smoking ( $60 \pm 3 \%$ relative humidity, $22 \pm 1^{\circ} \mathrm{C}$ ) according to ISO 3402:1999 [26]. Prior to smoking, three quality checks were performed on the RM20S as follows: the air velocity was verified to be $20 \pm 3 \mathrm{~cm}^{3}$ / sec; checks were performed for air leaks (acceptable range of pressure between 2750 and $3000 \mathrm{~Pa}$ ); and the puff volumes were adjusted on each syringe to $35 \pm$ $0.3 \mathrm{ml}[18]$.

\section{Syringe validation using hydrocarbon analysis}

To assess syringe accuracy and precision within the Borgwaldt RM20S, a total hydrocarbon analyzer - 3010P MINIFID Portable Heated Flame Ionisation Detection (FID) (Signal Group Ltd, Surrey, UK) was used to measure syringe output, as recommended by Borgwaldt. This FID has a stated accuracy of better than \pm 0.2 ppm, with linearity better than $\pm 0.05 \mathrm{ppm}$ for an analyte range of 0-100,000 ppm hydrocarbon, so was therefore well suited for this study as the measured $\mathrm{CH}_{4}$ concentrations ranged between $100-520$ ppm [18]. A $10 \%( \pm 0.5 \%)$ methane/nitrogen $\left(\mathrm{CH}_{4} / \mathrm{N}_{2}\right)$ test gas (Air Products PLC, Cheshire, UK) was diluted by all 8 syringes simultaneously, in the same way they would dilute cigarette smoke.

Each syringe output line was attached to a separate, vacuumed, 1.5 litre gas collection bag. After 9 minutes of syringe dilution and methane test gas collection (the 
length of time taken to fill a single bag) all bags were detached and the contents read using the hydrocarbon analyser. The analyser ignition fuel used was a mixture of hydrogen and helium gases $\left(40 \% \mathrm{H}_{2}, 60 \% \mathrm{He}\right.$ ) (Air Products PLC, Cheshire, UK). The analyser calibration gas was 500 parts per million (ppm) methane/air (CryoService Ltd, Worcester, UK).

The contents of each bag were recorded as ppm of the total methane test gas collected, and these were compared to a target ppm value for the dilutions used. Two dilutions were tested alternately to reduce mechanical bias, 1:500 and 1:193 (gas:air, v/v); $\mathrm{n}=15$ was the data set for each. The dilutions of 1:500 and 1:193 (a calibration standard dilution) were selected as this represents a low and mid dose of smoke respectively in the H292 cell line biological dose response range; the typical range being 1:1000 - 1:20 [17]. Methane target values for 1:500 and 1:193 were $200 \mathrm{ppm}$ and $520 \mathrm{ppm}$ respectively. Between alternate dilutions, gas collection bags were vacuumed with a pump and a clearing puff was initiated on the RM20S to clear all syringes and tubing of residual gas.

\section{Smoke transfer efficiency using an electrical mobility spectrometer}

To assess the extent of whole smoke loss/deposition within the tubing of the Borgwaldt RM20S smoking machine, an electrical mobility spectrometer - Differential Mobility Spectrometer 500 (DMS) (Cambustion Ltd, Cambridge, UK) was used. The DMS sampled all the smoke per puff reaching the sampling port over all 10 puffs used for assessment and recorded the average particle diameter, number and volume concentration, flow rate and time period of each sample to calculate the total number and volume of particles at different regions along the tubing.

The same single syringe of the RM20S and the same exposure chamber were used in combination for all readings to reduce inter-syringe variability and syringechamber pairing bias. Samples for aerosol analysis were taken at three points along its length: $(\alpha)$ the syringe exhaust line; $(\beta)$ pre-exposure chamber and $(\gamma)$ postexposure chamber (Figure 4). Readings at these three key points along the tubing were used to quantify, qualify and calculate smoke losses within the system.

The amount of smoke at the point of generation could not be measured directly in the RM20S smoking machine as there was no suitable access point - the earliest accessible point was $\alpha$, the exhaust port of the first syringe between four and six seconds after puff initiation. Measurement at the generation point was estimated by smoking the same cigarettes (3R4F) and measuring using the same DMS electrical mobility spectrometer. In this case a smoking cycle simulator (SCS)
(Cambustion Ltd, Cambridge, UK) was used as the smoking engine at the ISO regime allowing direct sampling over zero to two seconds from puff initiation via a constant flow sampling system [27]. These primary data were used to calculate subsequent smoke losses within the system.

The DMS was set to a secondary dilution of 1:60 to take $\alpha$ readings, as the smoke from the syringe at this stage was concentrated, but no secondary dilution for the $\beta$ and $\gamma$ readings was required. For $\gamma$ readings the chamber was filled with $30 \mathrm{ml}$ Dulbecco's Modified Eagles Medium (DMEM) (Fisher Scientific UK Ltd, Loughborough, UK) to mimic usual exposure conditions and to give the correct internal volume and humidity; media-in and media-out ports were blocked. A tap was also inserted between the DMS line and the chamber for the $\gamma$ readings, and was only opened for 8 seconds to sample aged smoke when the syringe was exhausting fresh smoke into the chamber; this stopped the DMS pump continually pulling smoke from the syringe line through the chamber and causing a vacuum. This is representative of whole smoke exposures to in vitro cultures in our laboratories using the RM20S.

A three-way comparison of different types of tubing was conducted on the RM20S to assess their individual performance and to see if there was a significant reduction in smoke loss using one type of tubing over another. The three tubing types compared were: existing polyurethane (the current used tubing installed in the RM20S upon manufacture, and having a visible build up/conditioning of tar), unused polyurethane (Farnell, Leeds, UK) and unused Viton ${ }^{\circledR}$ (Altec Products Ltd, Cornwall, UK), a PTFE-like fluoroelastomer. The tubing type order and the order in which the position was read along the tubing was fully randomised and repeated three times. The length of tubing used for each smoke run was $3.4 \mathrm{~m}$ irrespective of tubing type. For unused Viton ${ }^{\circledR}$ and unused polyurethane, new lengths of tubing were installed for each repeat experiment; when conditioned polyurethane was removed for another type of tubing, it was retained and later reinstalled for repeat experiments.

\section{Deposition assessment using fluorometric analysis}

Particle deposition within the whole smoke exposure chamber was determined by exposing inserts without cells to 3R4F reference cigarette smoke at a range of dilutions, minimum of $\mathrm{n}=4$ per dilution. Before smoke exposure, blank inserts were pre-wetted with deionised water and placed into the chambers. Whole smoke was generated and delivered as previously described. Deposited particulate material was extracted from inserts using $2 \mathrm{ml}$ of high performance liquid chromatography (HPLC) grade methanol (Hayman Ltd, Essex, UK) with 
agitation on a plate shaker set to $150 \mathrm{rpm}$ for $10 \mathrm{~min}$ utes. This procedure was repeated using a blank/ untreated insert as a control.

Extracts were analysed by HPLC using an Agilent 1100 Series (Agilent, UK). Elution was performed isocratically with a methanol flow rate of $1 \mathrm{ml} /$ minute and a sample injection volume of between 3-10 $\mu \mathrm{l}$. Fluorescence was detected with an Agilent standard FLD cell (Agilent, UK) at excitation and emission wavelengths of $286 \mathrm{~nm}$ and $350 \mathrm{~nm}$ respectively. Particulate concentrations of the insert extracts were calculated from the standard calibration curves and the blank insert results subsequently subtracted from the extract values. ChemStation Rev.A.10.02 (Agilent, UK) software was used to undertake instrument control and quantification. Sample extracts were quantified against an external standard prepared from 3R4F generated PM [17]. The standard calibration curve was prepared from PM concentrations ranging from $0.48-38 \mu \mathrm{g} / \mathrm{ml}$.

\section{Neutral Red Uptake}

After whole smoke exposure, cells were transferred to complete Ultraculture (Lonza Biologics Plc, Tewkesbury, UK) containing $2 \mathrm{mM}$ glutamine, $50 \mathrm{U} / \mathrm{ml}$ penicillin and $50 \mu \mathrm{g} / \mathrm{ml}$ streptomycin (Invitrogen, Paisley, UK) and incubated at $37^{\circ} \mathrm{C}, 5 \% \mathrm{CO}_{2}$ for a 24 hour recovery period. After incubation, the Neutral Red Uptake (NRU) assay was performed to assess cell viability in each insert as previously described, based on the National Institutes of Health [28].

Briefly, media was removed from each insert and replaced with $50 \mu \mathrm{g} / \mathrm{ml}$ Neutral Red solution (SigmaAldrich Company Ltd, Dorset, UK) for 3 hours, to allow for the active uptake of dye into viable cells. Inserts were washed twice in pre-warmed phosphate buffered saline (Fisher Scientific UK Ltd, Loughborough, UK) to remove any residual dye. After washing, cells were lysed with $500 \mu \mathrm{l}$ destain (50\% ethanol, $49 \%$ distilled $\mathrm{H}_{2} \mathrm{O}, 1 \%$ glacial acetic acid). Aliquots of $100 \mu \mathrm{l}$ elutate were read on a Multiskan Ascent Microplate Reader (Thermo Labsystems, Waltham, MA, USA) at a wavelength of 540 $\mathrm{nm}$ with a reference filter of $630 \mathrm{~nm}$. Neutral Red blanks were also included to remove background levels of dye absorbed by the inserts. Viability of exposed cells was calculated as a percentage of air controls.

\section{Statistics}

Data were reported as a mean \pm standard deviation. For syringe validation, a two-way ANOVA was used to compare the syringe outputs at the two dilutions tested, Dunnet's test was used to compare results to target and look at bias, and a Total Gage R\&R test was used to assess repeatability and reproducibility of all syringes at both dilutions, data set $\mathrm{n}=15$ for both dilutions. For transfer efficiency data, a General Linear Model (with Tukey's test) was used to test for differences in sample point and tubing type, data set $\mathrm{n}=3$. A one-way ANOVA was used to assess the differences in cell viability measured during the exposure chamber dosimetry study, data set $\mathrm{n}=20$. Statistical analysis for the above investigations was conducted using MINITAB ${ }^{\circledR}$ v.15.1.30 statistical software. All residual plots for all graphs were checked to ensure the quality of the data obtained. A pvalue $<0.05$ was considered statistically significant. Deposition analysis using fluorescence was conducted on GraphPad Prism ${ }^{\circledR}$ v.5.02, data set was at least $n=4$ for all dilutions tested and reported as a mean \pm standard error of mean.

\section{Conclusion}

In conclusion, we present a reliable and repeatable whole smoke exposure system consisting of a Borgwaldt RM20S smoking machine and BAT's exposure chamber. We are confident this system can be used to expose in vitro ALI cultures to eight independent and simultaneous doses of whole smoke knowing that smoke delivery, dilution precision, and chamber dose are reproducible and expected. This study further supports our previous study comparing syringe validation using $\mathrm{CH}_{4}$ measurements in two different laboratories; one being in the UK, the other in Canada [18].

This system will enable us to investigate the effects of physiologically relevant whole cigarette smoke doses on endpoints known to be relevant to major smoking related disease, namely cancer and chronic obstructive lung disease (chronic bronchitis and emphysema). Furthermore, these results can be used to support and enable quantification and alignment of whole smoke doses with human dosimetry data; recent studies within Group Research and Development (GR\&D) BAT have modelled and quantified regional tissue deposition of smoke within the different compartments of the respiratory tract [29].

We propose to further use this exposure system for the evaluation of future tobacco products designed to reduce the harmful effects of smoke and to investigate individual components of cigarette smoke. Furthermore, this system could be a useful alternative in vitro method for evaluating the effects of other aerosols and gaseous mixtures such as air pollutants, inhaled pharmaceuticals and cosmetics, and to examine occupational exposure scenarios.

\section{Acknowledgements}

The authors would like to thank David Thorne for developing and assisting in the initial experiments using hydrocarbon analysis; Jessica Perkins and Martin Arnold for their technical support on the deposition assessment using an electrical mobility spectrometer; Liz Pearce for the HPLC analysis; 
and Mark Barber at Borgwaldt KC for his engineering and mechanical expertise.

\section{Authors' contributions}

JA carried out cell culture, whole smoke exposure, cytotoxicity assays, syringe validation, smoke transfer efficiency studies and drafted the manuscript. DA performed fluorometric deposition assessments. GA participated in the experimental design of the study and performed statistical analysis. CD and JMCA participated in the design, operation and data analysis of smoke transfer efficiency studies. MG conceived the study, participated in its design and coordination and drafted the manuscript. All authors read and approved the final manuscript.

\section{Competing interests}

The authors declare that they have no competing interests.

Received: 6 May 2011 Accepted: 26 August 2011

Published: 26 August 2011

\section{References}

1. US Department of Health and Human Services: How tobacco smoke causes disease: the biology and behavioral basis for smokingattributable disease. A report of the Surgeon General. Atlanta; 2010 [http://www.surgeongeneral.gov/library/tobaccosmoke/report/full_report. pdf].

2. Van der Vaart H, Postma DS, Timens W, Ten Hacken NHT: Acute effects of cigarette smoke on inflammation and oxidative stress: a review. Thorax 2004, 59:713-21.

3. Lestari F, Markovic B, Green AR, Chattopadhyay G, Hayes AJ: Comparative assessment of three in vitro exposure methods for combustion toxicity. App Tox 2006, 26:99-114.

4. Winkler AR, Nocka KH, Sulahian TH, Kobzik L, Williams CM: In vitro modeling of human alveolar macrophage smoke exposure: enhanced inflammation and impaired function. Exp Lung Res 2008, 34:599-629.

5. Faux SP, Tai T, Thorne D, Xu Y, Breheny D, Gaça MD: The role of oxidative stress in the biological responses of lung epithelial cells to cigarette smoke. Biomarkers 2009, 14:90-6.

6. Johnson MD, Schilz J, Djordjevic MV, Rice JR, Shields PG: Evaluation of in vitro assays for assessing the toxicity of cigarette smoke and smokeless tobacco. Cancer Epidemiol Biomarkers Prev 2009, 18:3262-304.

7. Thorne D, Wilson J, Kumaravel TS, Massey ED, McEwan M: Measurement of oxidative DNA damage induced by mainstream cigarette smoke in cultured NCl-H292 cells. Mutation Research 2009, 673:3-8.

8. Haswell LE, Hewitt K, Thorne D, Richter A, Gaça MD: Cigarette smoke total particulate matter increases mucous secreting cell numbers in vitro: a potential model of goblet cell hyperplasia. Toxicol In Vitro 2010, 24:981-7.

9. Health Canada: Official Method T-503- In vitro micronucleus assay for mainstream tobacco smoke Ottawa: Health Canada; 2004

10. International Organization for Standardisation: Cigarettes - determination of total and nicotine-free dry particulate matter using a routine analytical smoking machine ISO 4387; Geneva, Switzerland: International Standards Organisation; 1991

11. Misra M, Leverette RD, Hamm JT, Vulimiri SV: In vitro toxicological evaluation of cigarette smoke particulate phase matter: effect of dimethyl sulfoxide as solvent. Cont to Tob Res 2010, 24:1-9.

12. Keith $\mathrm{CH}$, Tesh PG: Measurement of the total smoke issuing from a burning cigarette. Tobacco Science 1965, 9:61-64

13. Nakayama T, Kaneko M, Kodama M, Nagata C: Cigarette smoke induces DNA single strand breaks in human cells. Nature 1985, 314:462-464.

14. Cantrall DE, Sisson JH, Veys T, Rennard SI, Spurzem JR: Effects of cigarette smoke extract on bovine bronchial epithelial cell attachment and migration. Am J Physiol 1995, 268:L723-L728.

15. Bernhard D, Huck CW, Jakschitz T, Pfister G, Henderson B, Bonn GK, Wick G: Development and evaluation of an in vitro model for the analysis of cigarette smoke effects on cultured cells and tissues. J Pharm Toxicol Meth 2004, 50:45-51.

16. In vitro exposure of cells to smoke at the air liquid interface. CORESTA 2005 [http://www.coresta.org/Reports/

IVT_TF_Report_Smoke_Air_Liquid_Interface.pdf], Accessed 28 February 2011.
17. Phillips J, Kluss B, Richter A, Massey E: Exposure of bronchial epithelial cells to whole cigarette smoke: assessment of cellular responses. Altern Lab Anim 2005, 33:239-248.

18. Kaur N, Lacasse M, Roy JP, Cabral JL, Adamson J, Errington G, Waldron KC, Gaça MD, Morin A: Evaluation of precision and accuracy of the Borgwaldt RM20S smoking machine designed for in vitro exposure. Inhal Toxicol 2010, 22:1174-83.

19. Borgwaldt, Smoking Machine RM20S. 2010 [http://www.borgwaldt.de/ cms/borgwaldt-kc/produkte/rauchmaschinen/rotierende/rauchmaschinebrrm20s.html], Accessed 28 January 2011.

20. Scian MJ, Oldham MJ, Kane DB, Edmiston JS, McKinney WJ: Characterization of a whole smoke in vitro exposure system (Burghart Mimic Smoker-01). Inhal Toxicol 2009, 21:234-243.

21. Vitrocell Systems: Solutions for cultivation and exposure in vitro Smoking Robots/VC 10 features. [http://www.vitrocell.com/index.php? Nav_Nummer=5\&SubNav_Nummer=12\&R], Accessed 28 March 2011.

22. Aufderheide $M$, Knebel JW, Ritter D: An improved in vitro model for testing the pulmonary toxicity of complex mixtures such as cigarette smoke. Exp Toxicol Pathol 2003, 55:51-57.

23. Bombick E, Ayres PH, Doolittle DJ: Cytotoxicity assessment of whole smoke and vapour phase of mainstream and sidestream cigarette smoke from three Kentucky reference cigarettes. Tox Met 1997, 7:177-190.

24. Adam T, McAughey J, McGrath C, Mocker C, Zimmermann R: Simultaneous on-line size and chemical analysis of gas phase and particulate phase of cigarette mainstream smoke. Anal Bioanal Chem 2009, 4:1193-1203.

25. Supplier Quality Requirements Taskforce, Daimler Chrysler Corporation, Ford Motor Company, General Motors Corporation: Measurement systems analysis, reference manual. Michigan, United States: General Motors; 3 2002

26. International Organization for Standardisation: Tobacco and tobacco products - Atmosphere for conditioning and testing ISO 3402: Geneva: Switzerland: International Standards Organisation; 1999.

27. McGrath C, Warren N, Biggs P, McAughey J: Real-time measurement of inhaled and exhaled cigarette smoke: Implications for dose. Journal of Physics: Conference Series 2009, 151:1-012018.

28. National Toxicology Programe NIH Publication Number 01-4500 Guidance document on using in vitro data to estimate in vivo starting doses for acute toxicity. National Institute of Health; 2001 [http://iccvam. niehs.nih.gov/methods/acutetox/inv_cyto_guide.htm]

29. Dickens C, McGrath C, Perkins J, Zhuravskaya A, Biggs P, McAughey J: Measuring the regional deposition of tobacco smoke in the human respiratory system. CORESTA Congress, Edinburgh, Scotland; 2010, [abstract].

doi:10.1186/1752-153X-5-50

Cite this article as: Adamson et al: Assessment of an in vitro whole cigarette smoke exposure system: The Borgwaldt RM20S 8-syringe smoking machine. Chemistry Central Journal 2011 5:50.

Publish with ChemistryCentral and every
scientist can read your work free of charge
"Open access provides opportunities to our
colleagues in other parts of the globe, by allowing
anyone to view the content free of charge."
W. Jeffery Hurst, The Hershey Company.
- available free of charge to the entire scientific community
- peer reviewed and published immediately upon acceptance
- yours - you keep the copyright
Submit your manuscript here:
http://www.chemistrycentral.com/manuscript/

